\title{
THE REGULATORY EFFECTS OF PUR $\alpha$ ON PARP1 GENE EXPRESSION AND ITS FUNCTIONS ON DNA REPAIR
}

\author{
Wenxin $\mathrm{He}^{1 \uparrow}$, Yongling $\mathrm{Li}^{29}$, Xiaoguang Shi ${ }^{1}$, Juan Chai ${ }^{1}$, Bingying Zhang ${ }^{2}$, Shanshan Guo ${ }^{1 \#}$, Chengmin Yuan ${ }^{1}$, \\ Ping $\mathrm{Li}^{2}$, Tao Sun ${ }^{1}$ and Jianqi Cui ${ }^{1,2}{ }^{*}$ and Huichen Wang ${ }^{3 *}$
}

Author information: ${ }^{1}$ Ningxia Key Laboratory of Cerebrocranial Diseases, The Incubation Base of National Key Laboratory, Ningxia Medical University, Yinchuan, China; ${ }^{2}$ Department of Biochemistry and Molecular Biology, School of Basic Medical Sciences, Ningxia Medical University, Yinchuan, China; ${ }^{3}$ Chancellor' s Research Initiative (CRI)Radiation Institute for Science and Engineering (RaISE), College of arts and science, Prairie View A\&M University, Prairie View, USA

\begin{abstract}
A The luciferase reporter construct, which contains a polyADP-ribose polymerase 1 gene promoter, was transfected into U87MG cells with the Pura eukaryotic expression vector, and the activity of the PARP1 promoter was assessed by luciferase assay to evaluate the regulatory effects of Pura on PARP1 gene expression. The Pura eukaryotic expression vector was transfected into U87MG cells, and the cell total RNA and protein were extracted to determine the effects of Pura on PARP1 gene expression at transcriptional and translational levels by real time PCR and Western blot assay. The results demonstrated that Pur $\alpha$ can positively regulate PARP1 promoter activity and promote PARP1 gene expression both at transcriptional and translational levels. Further study illustrated that Pura can collaborate with PARP1 in the repair of damaged DNA; the results of a pull-down assay suggested that there is a physical interaction between Pura and PARP1. The overexpression of Pura can increase endogenous PARP1 expression and alleviate the expression of the DNA damage signal protein, $\mathrm{\gamma H} 2 \mathrm{AX}$. Above all, we believe that Pura possesses a positive regulatory effect on PARP1 gene expression and collaborates with PARP1 to repair damaged DNA.
\end{abstract}

Keywords: Pura eukaryotic expression vector, PARP1 promoter, PARP1 gene expression, damaged DNA

INTRODUCTION The maintenance of genomic DNA integrity is essential for cells to exert their normal functions. Exogenous factors such as ionizing radiation, UV light, and chemotherapeutic agents, can affect the integrity of the genetic materials. Endogenous processing, including oxidative metabolism, stalled DNA replications, and $V(D) J$ recombination also produce DNA damage that disrupts the integrity of the genetic materials. These genotoxic agents/events can create single-stranded DNA breaks (SSBs) or double-stranded DNA breaks (DSBs). Without timely and accurate repair, DSBs represent a

\footnotetext{
\# Current address: Shanshan Guo, Current address: San Quan College of Xinxiang Medical University, West of Changjiang Avenue, Pingyuan New Area, Xinxiang City, Henan Province, China.

*Corresponding author: Jianqi Cui, E-mail: jianqi@gmail.com (JQ.C); Huichen Wang, E-mail: huichenwang@yahoo.com (HC.W)

"These authors contributed equally to this work.

Wenxin He, Yongling Li, Xiaoguang Shi, Juan Chai, Bingying Zhang, Shanshan Guo, Chengmin Yuan, Ping Li, Tao Sun and Jianqi Cui and Huichen Wang (C)
}

critical injury for cells that will result in gene mutations and genomic instability, as well as the loss or recombination of cellular chromatin DNA. The changes caused by the DSBs lead to cell apoptosis or oncogenesis [1]. Much attention is currently being paid to the reaction of cells to the DNA damage in proliferating cells and to tumor development; ${ }^{[1]}$ however, comparatively little is known about the detailed effects of these processes on the nervous system. A proper cellular signaling reaction in response to DNA damage and the capacity to repair the damage is essential for the life of all organisms. Cells have a variety of DNA repair pathways to protect against damage [2].

The genes associated with DNA repair belong to the category of susceptibility genes, and polymorphism of these genes is common in organisms. This polymorphism can change the ability of individuals to repair DNA damage, and a decline or deficiency of repair ability may lead to instability of genomic DNA or oncogenesis. DNA damage-induced neurotoxicities have been associated 
with the pathogenesis of many degenerative diseases of the nervous system, such as Parkinson's disease, Alzheimer's disease, Huntington's disease, and amyotrophic lateral sclerosis. The key point at which the DNA damage participated in the genesis and development of the disorders and the mechanisms involved remain unclear [3]. In addition, recent studies have demonstrated that variants and polymorphisms of repair genes associated with DSBs, such as Ku80, RAD51, XRCC5, and XRCC1, are closely associated with high risks for oral cancer, glioma, hepatocellular carcinoma, and colorectal cancer [4-7]. A deficiency in the expression or the inactivation of an expressed product can also induce the instability of genomic DNA and is considered as the main reason for the genesis of breast cancer [8]. Poly(ADPribose) polymerase-1 (PARP1) is a nucleic enzyme that is located in the nucleus of eukaryotic cells and catalyzes polyADP-ribose polymerization. PARP1 is the main isoform of the 18 PARP family members. It consists of 1014 amino acids and has a molecular weight of $116 \mathrm{kDa}$. The encoding gene is located on chromosome $1 \mathrm{q} 41 \sim q 42$ and is composed of 23 exons, with an isoelectric point of $8.0 \sim 9.8$ [9]. PARP1 is a highly conserved intra-nuclear DNA binding protein and can recognize a low level of DNA damage, activate the DNA repair system, and repair damaged DNA. When DNA damage is very serious, PARP1 can initiate cellular apoptosis to maintain the integrity of the genomic materials of the cell [10].

Since ADP-ribose polymerization can regulate the reaction to DNA damage by inhibiting the PAR induced by DNA damage, it is more sensitive to genetic toxicity for tumor cells, especially tumor cells with a deficiency in DNA repair. Therefore, PARP inhibitors have been designed and tested in tumor treatment [11-13]. Thus far, the treatment effects have been less than satisfactory. For example, the efficiency in a phase II clinical experiment of BRCA-defective tumors was only $40 \%$ [12].

Pura is an omnipresent nucleic acid-binding protein with the ability to bind to specific DNA sequences, which were originally located on the promoter of the mouse myelin basic protein gene. Based on these characteristics, it was purified from mouse brain tissue [14-15]. The Pura sequence is highly conserved among the different species and there are only 2 out of 322 amino acid residues that differ between the mouse and human beings [16-18]. As a multifunctional protein, Pura plays important roles in many body functions, especially in neurogenesis and development. Our previous work demonstrated that Pura could negatively regulate amyloid precursor protein gene expression, which could indirectly decrease amyloid $\beta$ formation and might be helpful in the prevention of Alzheimer's disease. The function of Pura in the survival and differentiation of neuronal cells has been proven in transgenic mice [19] and a model of Pura gene knockout mice has also been successfully established. Pura knockout (Pura ${ }^{-/-}$) mice behaved normally at birth, but gradually developed neurological problems and displayed these abnormalities at the 15th postnatal day, which presented as serious tremors and unprompted seizures and finally death, inevitably by the 4th week after birth. Compared with the wild type age-matched littermates $\left(\right.$ Pur $\left.^{+/+}\right)$, the Pura ${ }^{-/-}$mice demonstrated abnormally small numbers of neurons in regions of the hippocampus and cerebellum, so it is not hard to speculate that Pura plays a critical role in neurogenesis. Abnormal chromosomes appeared in the Pura ${ }^{-/}$cells when the cells were treated with genotoxic reagents. This suggested that Pura played an important role in DNA repair during neurodegeneration [20]. In addition, Pura played a critical role in monitoring the repair of DSBs induced by stalled replication forks [21].

Based on the above observations, we designed the current experiment. DNA damage is a detriment to each type of cell. It has been reported that Pura and PARP1 contribute to DNA damage and repair, respectively, implying that an interaction between Pura and PARP1 exists in DNA damage and coordinates the process of repair. However, the relationship between Pura and PARP1 still remains unclear with respect to how this relationship is involved in DNA damage and repair. What intrigued us was that as a multifunctional protein, Pura could upregulate the gene expression of PARP1. These results have not been previously reported, so far as we know. We performed a series of experiments to investigate the mechanism of this relationship to produce theoretical support for further study.

\section{MATERIAL AND METHODS}

Prokaryotic expression vectors pGEX-4T-1 (GE Healthcare Lifesciences, Marlborough, USA) and pGEX-4T-1-Pur $\alpha$, eukaryotic expression vectors pCDNA3.0 (Invitrogen, USA) and pCDNA3.0-Pura, reporter vector pGL3-basic (Promega, USA), Hela cell line, and human glioma cell line U87MG were all grown in our laboratory. Pierce Glutathione Agarose and RevertAid First Strand CDNA Synthesis Kits were purchased from Thermo (USA). BugBuster ${ }^{\circledR}$ Maser Mix was purchased from Novagen (Germany). Commassie Blue Fast Staining Solution was purchased from Solarbio (China). IPTG was obtained from Merck (Germany). RT-PCR Mix was purchased from CWBIO (China). Topo pCR2.1 cloning kits, Opt-MEM 
medium, and lipofectamine2000 reagent were procured from Invitroge (USA). DMEM was obtained from HyClone (USA). Dual-Glo ${ }^{\circ}$ Luciferase Assay System was purchased from Promega (USA). A BCA kit was from Keygene Biotech (China) and Rabbit anti-Pura Polyclonal Antibody (ab79936) and Rabbit Anti- $\mathrm{H}_{2} \mathrm{AX}$ (phospho S139) Polyclonal antibody (ab2893) were purchased from Abcam (UK). Rabbit anti-PARP1 Polyclonal Antibody (TA332370S) was obtained from ORIGENE (USA). The Mouse Anti- $\beta$-actin monoclonal antibody (TA-09) was purchased from ZSBG-Bio Company (China).

\section{Luciferase reporter assay}

The control luciferase reporter plasmid, the constructed luciferase reporter plasmid for PARP1 pGL3-PARP1$500 /+100$, and the eukaryotic expression plasmid pCDNA3.0-Pura were transfected into U87MG cells together or separately. The U87MG cells were transfected with lipofectamine 2000 according to the manufacturer's instructions. At 48 hours after the transfection, cells were harvested for the experiment. In short, the culture medium was discarded, and the cells were washed with PBS, and then trypsinized. The trypsinized cell mix was

\begin{tabular}{lll}
\hline Primer & Sequence & Usage \\
\hline Primer1-F & 5'-CGGGG TACCG GAGGC & P PCR for \\
Primer1-R & TGAGG-3' & PARP1promoter \\
Primer4-F & 5'-CCGGA GCTCG AGCGG & PCR for \\
& CGGAG-3' & PARP1promoter \\
& 5'- AAGAC TCTGG GTGAC TTT-3’ & RT-PCR for PARP1 \\
Primer4-R & 5'-CACCA TCTTG GACAG-3' & RT-PCR for PARP1 \\
Primer4-F & 5'-CGTGT TTATG CGAGT GAG-3' & RT-PCR for Pur \\
Primer4-R & 5'-CCTCT GCTTC TCTTG AATC- & RT-PCR for Pur $\alpha$ \\
& 3' & \\
Primer-F & 5'-GAGTC AACGG ATTTG & RT-PCR for GAPDH \\
& GTCGT-3' & \\
Primer-R & 5'-GACAA GCTTC CCGTT & RT-PCR for GAPDH \\
& CTCAG-3' & \\
\hline
\end{tabular}

Table 1. Primers for PCR and RT-PCR

\section{Construction of a luciferase reporter plasmid containing the PARP1 gene promoter}

Using the PARP1 gene sequence, we designed primers for the amplification of the PARP1 gene promoter. The promoter spans from -500 to +100 , approximately 600 bps of the DNA sequence, on the proximal end of the PARP1 gene (Genbank, AL359704.9, 146087-146686). The primer sequences are listed in Table 1. To extract genomic DNA from U87MG cells, the synthesized primers were used to amplify the PARP1 promoter with high fidelity DNA polymerase $p f u$. We added deoxyadenosine to both ends of the amplified PCR fragment with taq DNA polymerase at $72^{\circ} \mathrm{C}$ for 30 minutes and then purified the fragment and ligated it with Topo pCR2.1 cloning vector. The positive colonies were selected using blue white screening and verified by sequencing. The appropriate fragment was cut with Kpn I and Xho I endonuclease and then purified and subcloned into the responsive MCS upstream of the luciferase gene in the reporter plasmid pGL3-Basic to construct the PARP1 promoter reporter plasmid pGL3Basic-PARP1-500/+100. spun at $1000 \mathrm{rpm}$ for 5 minutes to collect the cell pellets and then $100 \mu \mathrm{l} 1 \mathrm{X}$ lysis buffer was added to lyse the cells. The cell lysate was centrifuged at $15000 \mathrm{rpm}$ for 10 minutes and the supernatant was separated for the luciferase activity analysis. To measure the luciferase activities, $50 \mu \mathrm{l}$ of supernatant was added to 96-well plates. In addition, $50 \mu \mathrm{l}$ Dual-Glo ${ }^{\circ}$ Luciferase Reagent and Dual-Glo Stop \& Glo Reagent were added to each well and measured with a Centro LB 960. The luciferase assay was performed according to the instructions from Promega for the Dual-Glo ${ }^{\circ}$ Luciferase Assay System. The activities of Renilla were used as an internal reference to adjust for transfection efficiency.

\section{Real Time PCR (RT-PCR)}

The Pur $\alpha$ eukaryotic expression vector, pCDNA3.0-Pur $\alpha$, and the responsive control, pCDNA3.0, were transfected into U87MG cells with lipofectamine 2000. After the transfection (48 hours), the total cellular RNA was extracted with TRIzol. The extracted RNA was measured by a Nanodrop spectrophotometer to check the purity and quality. Reverse transcription was performed with a 
RevertAid First Strand cDNA Synthesis Kit. The synthesized cDNA solution was diluted 10 -fold, and $2 \mu$ were used for Real Time-PCR in a Bio-Rad CFX96 ${ }^{\mathrm{TM}}$ Real-Time System. The primers used for PCR were listed in Table 1, and GAPDH was used as an internal control.

\section{Western blot assays}

Pura eukaryotic expression vector, pCDNA3.0-Pur $\alpha$, SDS and the corresponding control, pCDNA3.0, were transfected into U87MG cells. After 48 hours, the cells were harvested for protein extract. The transfected cells were washed with ice-cold PBS and then lysed with RIPA buffer with protease inhibitor and phosphorylase inhibitor. The cell debris was removed by centrifugation and protein concentration was quantitated with BCA reagent. Furthermore, $40 \mu \mathrm{g}$ of total protein was taken for SDS-polyacrylamide gel electrophoresis (PAGE). The protein was transferred to a PVDF membrane and blocked with $5 \%$ dry milk blocking buffer. The primary antibody was added, and the membrane was incubated at $4^{\circ} \mathrm{C}$ overnight. The next day, the membrane was washed with TBST buffer 5 times ( 5 minutes each time); the second antibody was added and incubated at room temperature for 60 minutes, then washed again as described above. The membrane was detected with an ODYSSEY CLX imaging system. The dilution of the primary antibody was $1: 1000$, it was $1: 3000$ for the internal control antibody $\beta$ actin, and it was 1:5000 for the fluorescent-tagged second antibody.

\section{Pull down assay}

To transform the recombinant plasmid of GST-Pura and the GST vector into competent BL21 cells, they were plated onto agar plates that contained ampicillin, and incubated at $37^{\circ} \mathrm{C}$ overnight. On the second day, a single clone was collected and put into $5 \mathrm{~mL}$ LB medium and allowed to grow overnight. The next day the culture was expanded to $100 \mathrm{~mL} \mathrm{LB}$ medium for large-scale expression and purification of the target protein. Bacteria were incubated at $37^{\circ} \mathrm{C}$ in a shaking incubator until the culture had reached the mid-log phase of growth $\left(O D_{590}=0.6\right)$. The target protein's expression was induced with IPTG ( $2 \mathrm{mM}$ ) at $29^{\circ} \mathrm{C}$ and $220 \mathrm{rpm}$. After the induction, the cells were grown for 4 hours and harvested by centrifugation at 4000 $\mathrm{g}$ for 5 minutes. Lysis buffer was added to resuspend the cell pellet ( $5 \mathrm{ml}$ of lysis buffer per $100 \mathrm{ml}$ of cell culture). The target protein was extracted based on the protocol from Novagen. Subsequently, $500 \mu$ l of Pierce $^{\circledR}$ Glutathione Agarose was added to the protein extract. The mixture was shaken at $4^{\circ} \mathrm{C}$ overnight to adequately combine the target protein with Glutathione Agarose Beads. The mixture was centrifuged at $1000 \mathrm{rpm}$ for 3 minutes to precipitate the Glutathione Agarose Beads. The beads were washed 20 times with cold PBS and a small amount of the supernatant was saved to determine the protein concentration and to analyze by SDSpolyacrylamide gel electrophoresis. Coomassie Brilliant Blue Staining was performed to evaluate the purity of the protein. After purification, the beads binding the protein were used to pull down its interacting protein from HeLa whole cell extracts (WCE). HeLa WCE (1 mg) was precleared by GST beads and then bound with GST or GSTPura beads. Binding proteins were eluted with NTN buffer containing $500 \mathrm{mM} \mathrm{NaCl}$. The eluted proteins from GST beads and GST-Pura beads were separated on 4-20\% SDSPAGE, and GST-Pur $\alpha$ was loaded as a control. The gels were stained with GelCode blue stain reagent. Many protein bands were presented on the GST-Pura lane. The strongest stained band was analyzed by mass spectrometry. The protein band was digested by trypsin and the digested peptide was analyzed using a MALDIToF/Pro (Amersham Biosciences) mass spectrometer and matched to peptide sequence information.

\section{Statistical analysis}

All data reported are the mean \pm s.e.m $(\bar{x} \pm S)$ and were analyzed using IBM software SPSS (version 20). One-way ANOVA was used for analyses. LSD method was performed to compare the means between multiple groups. For all analyses, $\alpha=0.05$. Experiments were repeated at least 3 times.

\section{RESULTS}

\section{Construction of a Luciferase reporter plasmid containing the PARP1 gene promoter}

To identify the reporter plasmid, pGL3-PARP1, we used endonuclease cutting (Fig 1) and sequence analysis (Fig 2).

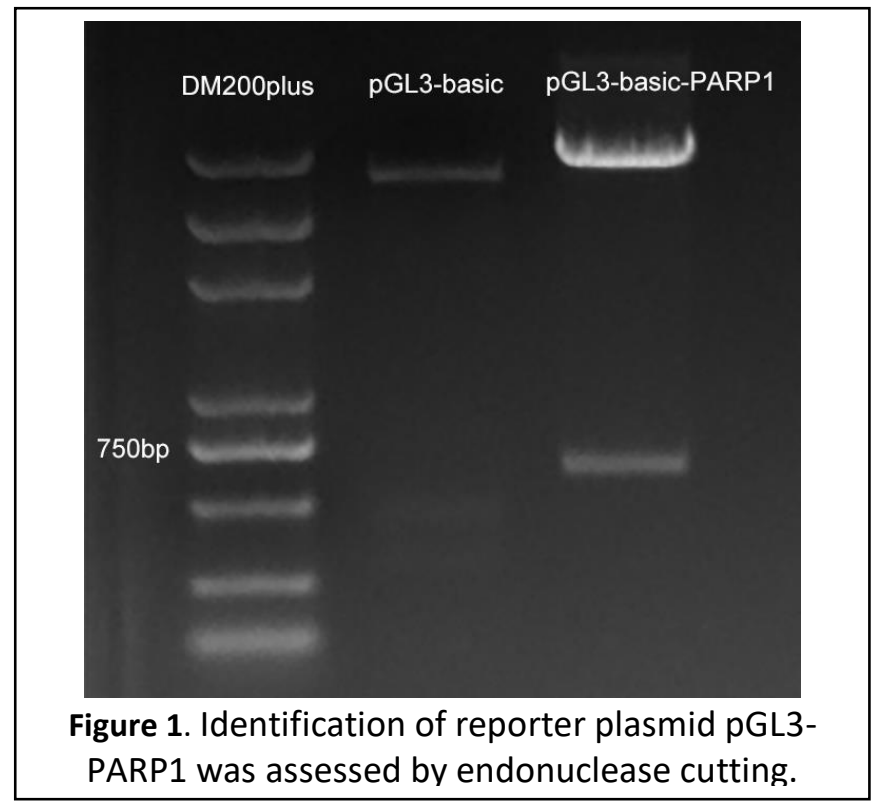


The band between $750 \mathrm{bp}$ and $500 \mathrm{bp}$ illustrated the band of $600 \mathrm{bp}$ that we successfully inserted into the reporter plasmid, pGL3-PARP1. Meanwhile, the sequencing result demonstrated that the inserted fragment was a PARP1 promoter. The results are consistent with our experimental design. According to the luciferase assay, the activity of the constructed plasmid was sufficient for the experimental requirement. much higher activity of the PARP1 gene promoter, which indicated that Pur $\alpha$ significantly upregulated the activity of the PARP1 promoter (Fig 3).

To check the effects of Pura on PARP1 gene expression at the transcriptional level with $\mathrm{qPCR}$

To further investigate the effects of Pura to regulate

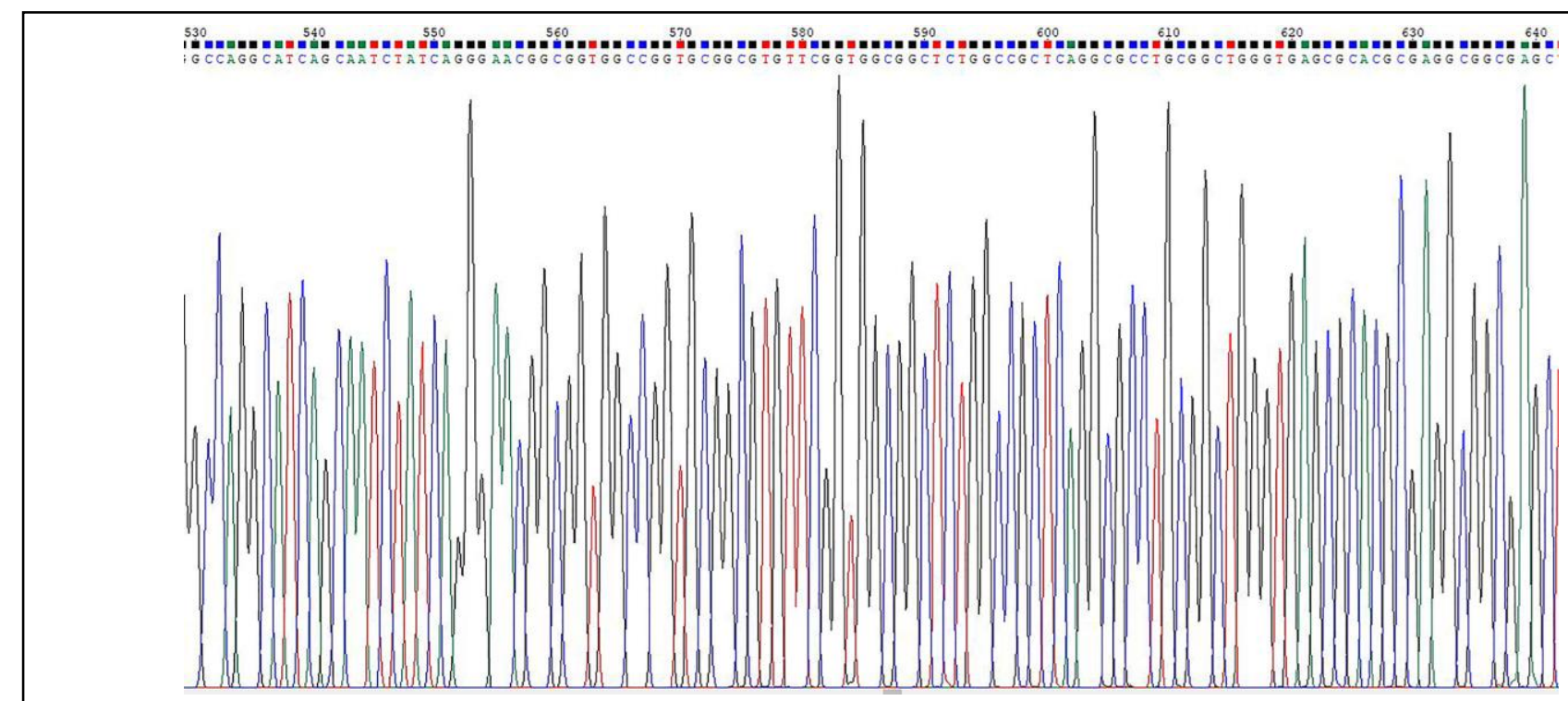

Figure 2. Identification of reporter plasmid pGL3-PARP1 was assessed by sequence analysis.

To evaluate the effects of Pura on PARP1 promoter activities with a Luciferase reporter assay

The constructed PARP1 reporter plasmid expressed great activity and was suitable for investigations of PARP1 gene expression. When transfected into U87MG cells, it had remarkable activity, while the empty reporter plasmid showed almost no activity with the luciferase assay. Pura illustrated up-regulatory effects on PARP1 gene expression when co-transfected PARP1 reporter plasmid in U87MG cells was compared with those co-transfected with pCDNA3 (control group). The Pura group expressed

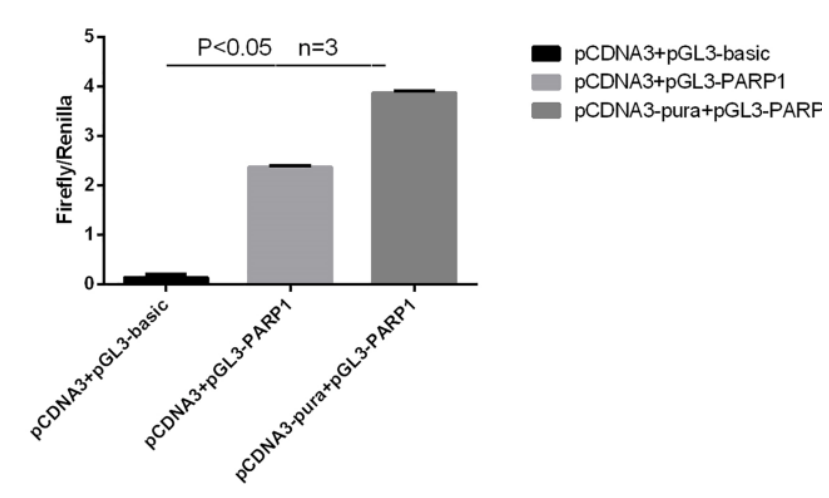

Figure 3. The effects of Pura on PARP1 promoter activity when cotransfected into U87MG cells.
PARP1 gene expression at the transcriptional level, U87MG cells were transfected with the eukaryotic expression plasmid, pCDNA3.0-Pura, and the control plasmid, pCDNA3.0. After the transfection (48 hours), the cellular total RNA was extracted and applied to the qPCR assay. The RNA contents of Pur $\alpha$ and PARP1 were evaluated with qPCR to confirm that the transfected Pur $\alpha$ had been overexpressed and to investigate the effects of Pura on PARP1 gene expression. The results of the qPCR demonstrated that compared with control group (pCDNA3) the content of PARP1 RNA in the Pura group increased remarkably. The results indicated that Pura significantly upregulated PARP1 gene expression, which is consistent with the results of the luciferase assay, which indicated that Pura promotes the expression of PRAP1 (Fig 4).

\section{Western blot assays}

To investigate the effects of Pura on regulation of PARP1 gene expression at the translational level, U87MG cells were transfected with the eukaryotic expression plasmid of pCDNA3.0-Pur $\alpha$ and the control plasmid, pCDNA3.0. The total cellular proteins were extracted and Western blot assays were performed. The results illustrated that 


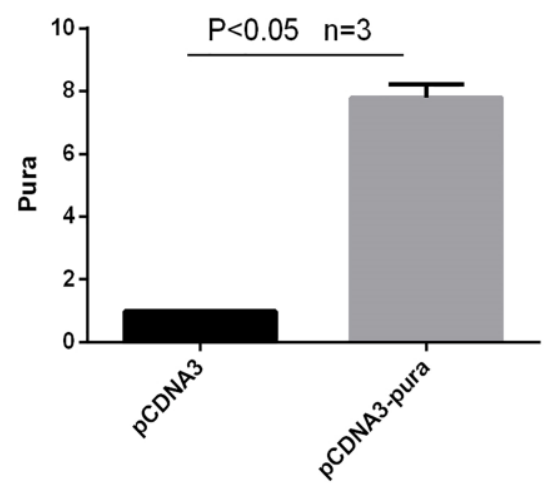

A
PCDNA3 pCDNA3-pura

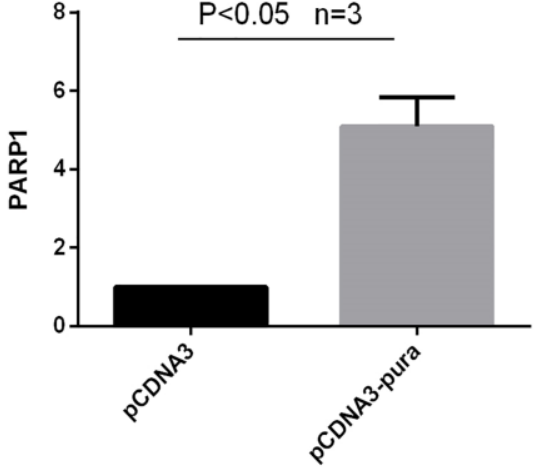

$\mathrm{B}$
pCDNA3

pCDNA3-pura

Figure 4. The effects of Pura overexpression on endogenous PARP1 gene expression.

A.

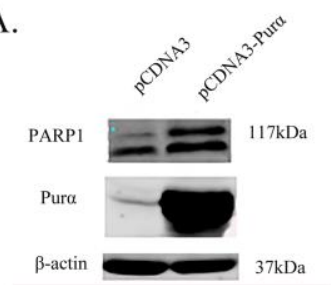

B.

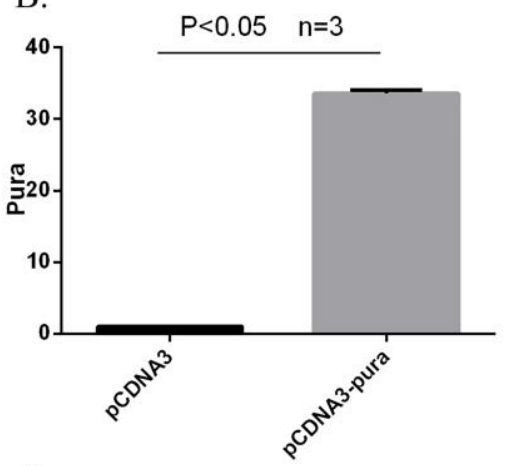

C.

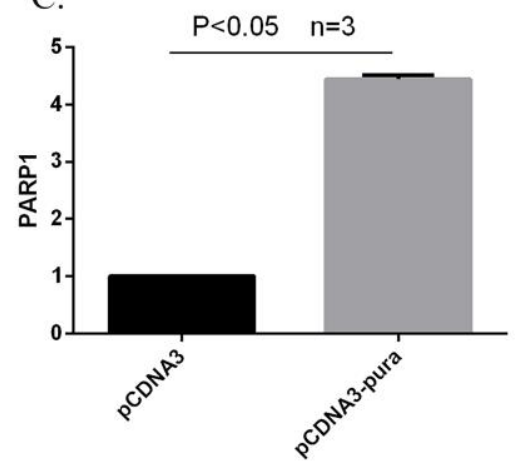

Figure 5. Western blotting analysis assessed the effects of Pura on PARP1 gene expression. (A) Western blotting assay to determine the effects of Pura overexpression on PARP1 gene expression. (B) (C) The statistical analysis results of Western blotting.
A.

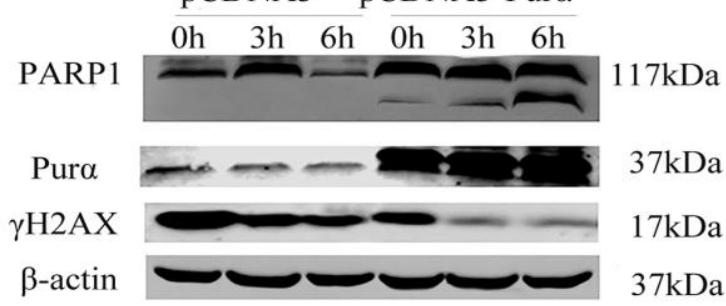

B.

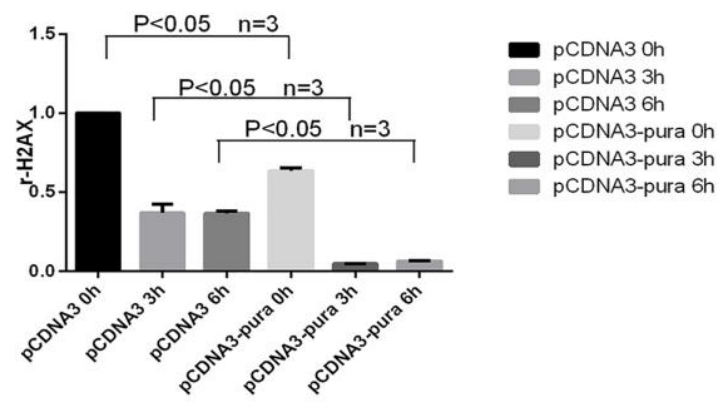

C.

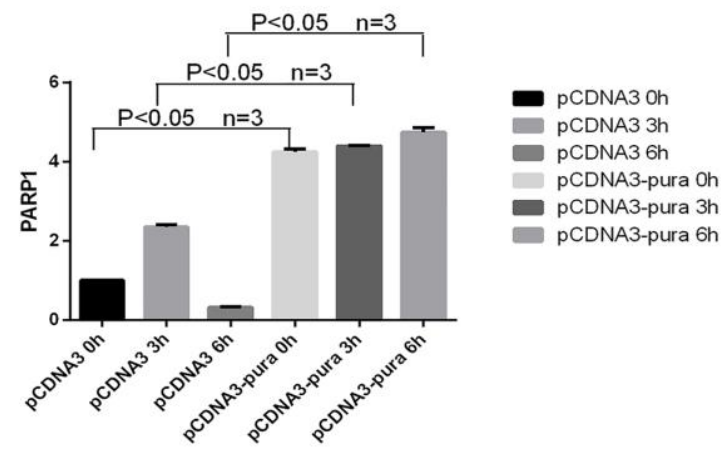

Figure 6. The effects of Pur $\alpha$ on PARP1 gene expression and alleviated the DNA damage. (A) The results of Western blotting show the effects of Pura on PARP1 gene expression within DNA damage; (B) (C) The statistical analysis results of Western blotting. 
Pura had a remarkably positive regulatory effect on the expression of PARP1 (Fig 5).

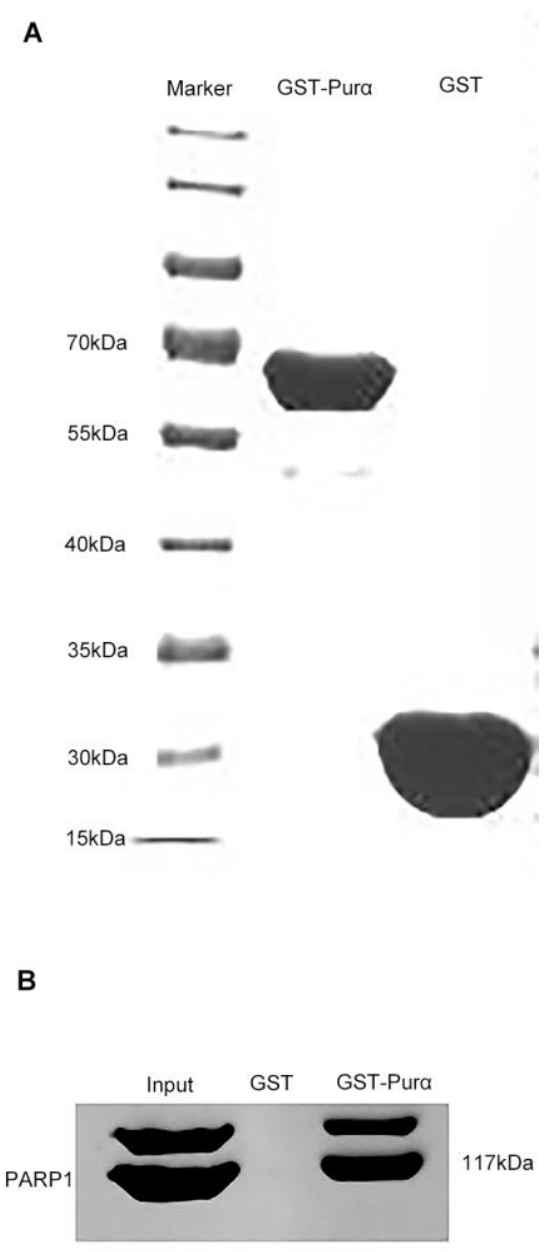

Figure 7. The results of purification of GST and GSTPura proteins.
In order to explore the interaction between Pur $\alpha$ and PARP1 in the context of DNA damage, hydroxyurea (HU) was used to build a model of DNA damage and then the expression of Pura and PARP1 were evaluated. To observe the dynamic changes in the DNA damage induced by $\mathrm{HU}$, the content of $\mathrm{\gamma H} 2 \mathrm{AX}$ at different time points was checked $(0 \mathrm{~h}, 3 \mathrm{~h}$, and $6 \mathrm{~h}$ after the $\mathrm{HU}$ treatment). The results indicated that after the $\mathrm{HU}$ treatment, DNA damage occurred and $\mathrm{yH} 2 \mathrm{AX}$ content increased dramatically at the $0 \mathrm{~h}$ time point. As time passed, the content of $\mathrm{pH} 2 \mathrm{AX}$ decreased. That meant that the repair system inside the cells had been awakened and the cells started to repair the damage. By the $6 \mathrm{~h}$ time point after the $\mathrm{HU}$ treatment, the content of $\mathrm{yH} 2 \mathrm{AX}$ returned to less than at the time of the $\mathrm{HU}$ treatment. Compared with the Pura overexpression group, the expression of PARP1 was much lower in the control group. On the contrary, the contents of $\mathrm{pH} 2 \mathrm{AX}$ was much higher in the control group than in the Pura overexpression group at each time point. Pura had a higher expression level in the overexpression group compared with the control group (Fig 6). The results demonstrated that $\mathrm{HU}$ could induce DNA damage and $\mathrm{YH} 2 \mathrm{AX}$ is a very sensitive early DNA damage signal protein. PARP1 was upregulated with Pura overexpression. With greater expression of PARP1 and higher levels of Pura expression, the content of $\gamma \mathrm{H} 2 \mathrm{AX}$ decreased, which indicated that Pura could up regulate the PARP1 gene expression and as a result, it alleviated the HU-induced DNA damage.

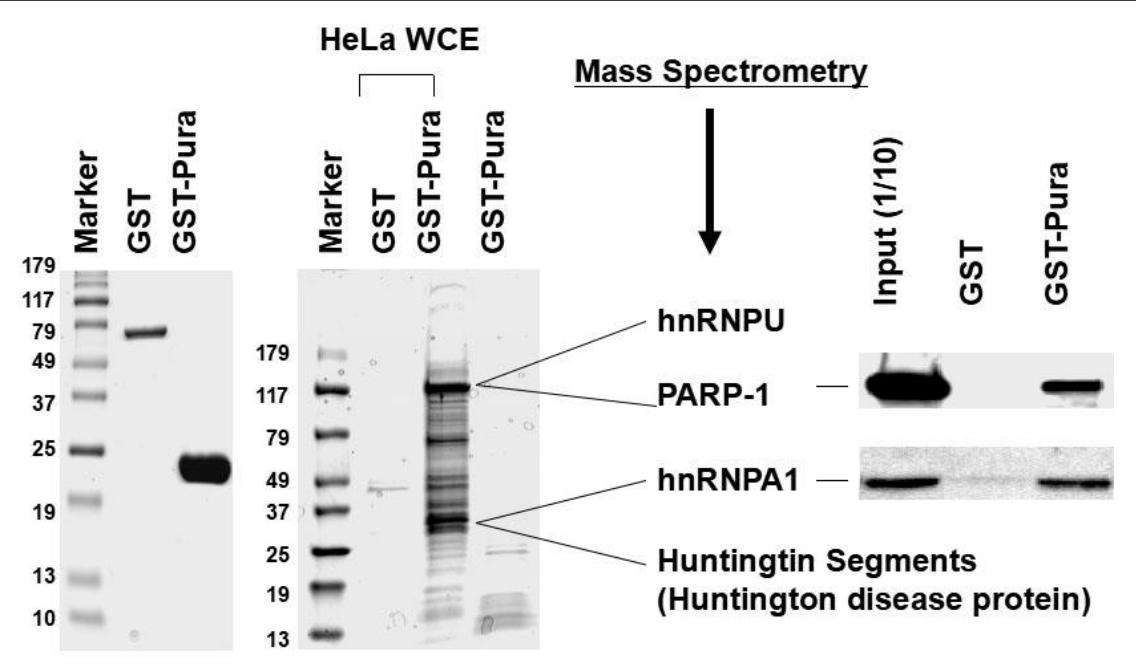

Figure 8. The interaction between Pura and PARP1 was assessed by pull down assay. 


\section{Pull down assay}

The GST fusion protein Pura and GST control were purified from in vitro induced prokaryote recombined bacteria. The protein was purified with glutathione sepharose beads and used as an immobile phase. The purified GST and GST-Pura were applied to polyacrylamide gel electrophoresis (PAGE) to check the purity of the proteins. After PAGE, the gel was stained with Coomassie Brilliant Blue Staining solution and then destained. The results demonstrated that purified GST was located at $26 \mathrm{kDa}$, while the fusion GST-Pura was located at $63 \mathrm{kDa}$ compared with the $37 \mathrm{kDa}$ for Pura (Fig 7). The sepharose beads linked protein was used to pull down HeLa cell whole extracts in order to identify the physical interaction with PARP1. The results (Fig 8) illustrated that Pura could pull down PARP1 protein from HeLa whole cell extracts. This indicated the presence of physical interactions between PARP1 and Pura.

DISCUSSION \& CONCLUSION Accumulating evidence suggests that PARP is a form of nucleic enzyme that is located in the nucleus of eukaryotic cells and catalyzes polyADP-ribose polymerization, and thus plays a crucial role in repairing and recognizing DNA damage, chromosome remodeling, the formation of the mitotic apparatus, and cell death. Over-activation of PARP is involved in many diseases. The main isoform, PARP1, is critical for DNA damage and repair functions. This function is also related to oncogenesis and chemotherapy resistance. Historical studies have identified 18 members of the PARP family, of which the most important and abundant is PARP1. PARP1 repairs SSBs via the BER pathway. Damaged DNA activates PARP, which cleaves $\mathrm{NAD}^{+}$into nicotine and ADP-ribose. The ADP-ribose then forms polyADP-ribose (PAR), combining with the receptor that contains PARP1, histones, and other proteins for DNA repair. Dissociation from PARP1 occurs at the DNA breaks when PARP1 is modified by PAR, which adheres to the breaks instead. The polymer has many negative charges, and thereby recruits major proteins in the BER-SSBS pathway, such as XRCC1. Other proteins that contribute to the spatial structural formation of chromosomes and DNA repair and replication are non-covalently bound to PAR, as well. These recruitment effects collaborate with noncovalent binding to exert DNA repair. Recent work has suggested that PARP is involved in more DNA damage and repair functions, such as recruiting MRE11 and ATM, inhibiting E2F4 and P130 compounds to influence the expression of BRCA1 and RAD51, ${ }^{[22]}$ and interacting with protein kinase complexes for the repair of DNA DSBs. Studies have shown that cells treated with alkylating agents, PARP1 inhibitors, or RNAi inhibited the expression of PARP1 and resulted in different consequences of DNA damage and repair. PAR can be catalyzed and synthesized by activated PARP1, which performs protein modification functions after translation in the cells.

A recent study confirmed the function of Pura in cell survival and differentiation in transgenic mice. Neuron numbers were decreased significantly in the hippocampus and cerebellum of young mice, which indicated that Pura played a crucial role in the development of neurons. The Pura (-/-) cell line showed chromosomal abnormalities when treated with genotoxic agents, which suggested that Pura was vital to DNA damage and repair in neurodegenerative diseases. Additionally, another study demonstrated that Pura executed surveillance in DSBs that was caused by replication fork halt. The polyribosome and hnRNP joined Pura in forming a complex that was enriched in the cytoplasm of neuronal cells, especially in the branch point of synapses. Pura also contributed to the stability of the genome and was exclusively expressed in specific tissues. A previous study confirmed that Pura mediated a specific mechanism of transport and translation, while others have found that a mutation in Pura can cause profound neonatal hypotonia, seizures, and encephalopathy in $5 q 31.3$ microdeletion syndrome. ${ }^{[23]}$ Hunt et al. revealed by whole exome sequencing in family trios that de novo mutations in Pura led to neurocognitive disorders, severe neurodevelopmental delays, and learning disabilities. ${ }^{[24]}$ Our study is the first that illustrated that Pura upregulated PARP1 gene expression. Our results demonstrated that when Pura was overexpressed, the PARP1 promoter activities were activated. Our qPCR and western blot results also confirmed that Pura could positively regulate PARP1 gene expression both at transcriptional and translational levels. Our experiment also found that when the Pur $\alpha$ gene was knocked out, the PARP1 expression level in mouse embryo fibroblast cells (Pura-/-MEFs) was much lower than in their wild type cells. We also found that re-transfected Puro genes in the Pur $\alpha-/-$ MEFs, can successfully reverse PARP1 gene expression (data not show). These results confirmed that Pura plays an important role in PARP1 gene expression. Although the detailed mechanisms for this up-regulation on PARP1 gene expression are unclear, as a transcriptional factor, Pura could be an important regulator of gene expression. The regulatory functions exhibited great variances and complexity. For some genes, Pur $\alpha$ exerted a positive regulatory effect, and acted as a transcriptional activator, such as the neuron-specific FE65 protein and PDGF-A. For other genes, Pura had a negative effect on transcription, 
such as with amyloid precursor protein. Pura also negatively regulated its own promoter activities. ${ }^{[21]}$

Using the pull-down assay, we found that some physical interactions existed between Pur $\alpha$ and PARP1. No similar reports have been previously published, to our knowledge. This physical interaction might explain how Pur $\alpha$ and PARP1 together exert the up-regulatory effects on the PARP1 gene expression, but a detailed view of the mechanism requires further investigation. Surprisingly, in the second band of the pull-down assay (Fig 8), we found heterogeneous nuclear ribonucleoprotein $\mathrm{A} 1$ and Huntingtin C-terminal segment (Huntington disease protein). The interaction of Pura with hnRNP A1 has been confirmed by western blot and immunoprecipitation (data not shown).

PARP1 is involved in the response to DNA damage. Our results showed that Pura is involved in DNA repair, probably by preventing and repairing DNA DSB formation at stalled replication forks. Pura is also associated with PARP1 to maintain poly(ADP-ribose) (PAR) homeostasis in the central nervous system. We also noted the effects of PARP1 on DNA damage and repair. The change in PARP1 after DNA damage was consistent with the previous work that demonstrated that $\mathrm{HU}$ was unable to inhibit the transcription of PARP1. Reports claim that in the early stages of cell damage, extracellular signals activate PARP1 through the MAPK pathway, a pathway involved in immune responses and inflammation. For instance, ROS activates PARP1 through the MAPK pathway to influence apoptosis.

The effects of Pur $\alpha$ and PARP1 on the development of the neuronal system have previously been described. Those studies focused on the protective effects of Pura on neurons, the contribution of PARP1 to SSBs, and the PARP1 inhibitor in cancer treatment. Few studies concentrated on the effects of Pura on the expression of PARP1. Here, we demonstrated that Pura regulates the function of PARP1, providing multiple lines of evidence for the influence of Pur $\alpha$ and the interaction between the two proteins. This relationship implies a possible route for anticancer drug development. Furthermore, we plan to perform immunofluorescence techniques to investigate the distribution of Pur $\alpha$ and PARP1 intracellularly in the damage model, and we plan to employ coimmunoprecipitation to study the status of these two proteins intracellularly. Next, we plan to construct various deletion mutants and nucleic acid-protein binding experiments, such as EMSA, to examine the binding point of Pura and the promoter of PARP1.

\section{REFERENCES:}

1. Goodarzi AA, Jeggo PA. The repair and signaling responses to DNA double-strand breaks. Advances in genetics 2013; 82: 1-45.

2. Williams B, Mancia G, Spiering W, AgabitiRosei E, Azizi M, Lip GYH, McManus R,Narkiewicz K, Ruschitzka F, Schmieder RE, Shlyakhto E, Tsioufis C, Desormais I; ESC Scientific Document Group. 2018 ESC/ESH Guidelines for themanagement of arterial hypertension. Eur Heart J. 2018 Sep 1;39(33):3021-3104. Doi: 10.1093/eurheartj/ehy339. PubMed PMID: 30165516.

3. Iadecola C, Yaffe K, Biller J, Bratzke LC, Faraci FM, Gorelick PB, Gulati M, Kamel H, Knopman DS, Launer L, Saczynski JS, Seshadri S, Zeki Al Hazzouri A. Impact of hypertension on cognitive function: a scientific statement from the American Heart Association. Hypertension 2016;68: e67.

4. Coca A, Monteagudo E, Domnech M, Camafort M, Sierra C. Can theTreatmentof Hypertension in the Middle-Aged Prevent Dementia in the Elderly?High Blood Press Cardiovasc Prev. 2016;23(2):97-104.

5. Mancia G., Fagard R., Narkiewicz $K$ et al. ESH/ESC Guidelines for the management of arterial hypertension The Task Force for the management of arterial hypertension of the European Society of Hypertension (ESH) and of the European Society of Cardiology (ESC) Eur. Heart J. 2013;34(28):2159-2219.

6. J. Jiménez-Balado, I. Riba-Llena. Cognitive Impact of Cerebral Small Vessel Disease Changes in Patients with Hypertension. Hypertension. 2019; 73:342-349. https://doi.org/10.1161/HYPERTENSIONAHA.118.120 90

7. Folstein M.F., Folstein S.E., McHugh P.R. Mini-Mental State: a practical guide for grading the mental state of patients for the clinician. J. Psych. Res. 2015; 12:189198.

8. Borson S, Scanlan JM, Watanabe J, Tu SP, Lessig MJ. Simplifying detection of cognitive impairment: comparison of the Mini-Cog and Mini-Mental State Examination in a multiethnic sample. Am GeriatrSoc, 2015; 53(5):871.

9. Milevs'ka-Vovchuk L.S. Comparative characteristics of screening scales for the detection of cognitive impairment. 2015;8(78):41-44.

10.Zaharov V.V. Neuropsychological tests: the need and feasibility. Consiliummedicum. 2011;12(2):98-106.

11.RubynshtejnS.Ja. Experimental methods of pathopsychology and experience in their application in the clinic. Study of verbal memory according to the method of Luria. A practical guide. Moscow; 2007. 
12.Armitage $P$, BerryG, Matthews JNS. (2002) Statistical Methods in Medical Research, 4th ed. Blackwell, Oxford.

13. Elias MF, Wolf PA, D'Agostino RB, Cobb J, White LR. Untreated blood pressure level is inversely related to cognitive functioning: the Framingham study. Am J Epidemiol. 2013; 138:353-364.
14.Gorelick PB, Scuteri A, Black SE, Decarli C, Greenberg $S M$, ladecola $C$, Launer $L$, Laurent $S$, Lopez $\mathrm{OL}$, Nyenhuis D, Petersen RC, Schneider JA, Tzourio C, Arnett DK, Bennett DA, Chui HC, Higashida RT, Lindquist R, Nilsson PM, Roman GC, Sellke FW, Seshadri S. Vascular contributions to cognitive impairment and dementia. Stroke 2011; 42:26722713. 